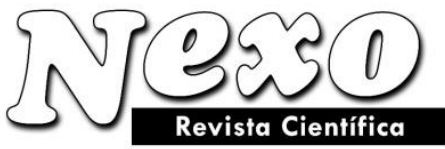

ISSN-E1995-9516

Universidad Nacional de Ingeniería COPYRIGHT @ (UNI). TODOS LOS DERECHOS RESERVADOS http://revistas.uni.edu.ni/index.php/Nexo https://doi.org/10.5377/nexo.v32i02.9265

\title{
DISEÑO TÉRMICO DE UN INTERCAMBIADOR DE CALOR DE DOBLE TUBO PARA EL ENFRIAMIENTO DE ACETONA
}

\section{THERMAL DESIGN OF A DOUBLE-TUBE HEAT EXCHANGER FOR ACETONE COOLING}

\author{
A. Pérez Sánchez ${ }^{1, *}$, E.J. Pérez Sánchez ${ }^{2}$ \\ ${ }^{1}$ Universidad de Camagüey "Ignacio Agramonte Loynaz", Facultad de Ciencias Aplicadas, Departamento \\ de Ingeniería Química, Camagüey, Cuba. \\ ${ }^{2}$ Empresa Servicios Automotores S.A., Dirección Comercial, Ciego de Ávila, Cuba. \\ *amauryps@nauta.cu
}

(recibido/received: 06-Agosto-2019; aceptado/accepted:15-Noviembre-2019)

\begin{abstract}
RESUMEN
El intercambiador de doble tubo es una de las piezas más simples de equipamiento en la cual se desarrolla el intercambio de calor de forma continua entre dos fluidos. En el presente trabajo se llevó a cabo el diseño térmico de un intercambiador de calor de doble tubo para efectuar el enfriamiento de una corriente de acetona líquida. También se evaluó la influencia del incremento del caudal de alimentación de la acetona sobre seis parámetros de diseño del intercambiador de calor: 1) coeficiente global de transferencia de calor; 2) área de transferencia de calor; caída de presión de las corrientes de 3) acetona y 4) etilenglicol; 5) calor intercambiado y 6) número total de horquillas necesarias. El intercambiador deberá poseer un área total de transferencia de calor de $6,0 \mathrm{~m}^{2}$ y número total de horquillas de 10 para cumplir con la demanda térmica del sistema. Tanto la corriente de acetona como de etilenglicol presentan valores de caída de presión inferiores al límite máximo permisible establecido por el proceso (110 $000 \mathrm{~Pa})$. El incremento del caudal de alimentación de la acetona ocasiona el aumento de los seis parámetros estudiados.
\end{abstract}

Palabras claves: Caída de presión; Diseño; Horquillas; Intercambiador de calor.

\begin{abstract}
The double-tube heat exchanger is one the simplest piece of equipment which performs heat exchange in a continuous mode between two fluids. In the present work the thermal design of a double-tube heat exchanger for the cooling of a liquid acetone is carried out. It was also evaluated the influence of the increment of acetone feed flowrate over six design parameters of the heat exchanger: 1) overall heat transfer coefficient; 2) heat transfer area; pressure drop of 3) acetone and 4) ethylene glycol streams; 5) heat exchanged and 6) total amount of hairpins needed. The heat exchanger must have a total heat transfer area of $6.0 \mathrm{~m}^{2}$ and a total amount of hairpins of 10 to fulfill the system's thermal duty. Both the acetone and ethylene glycol streams have pressure drop values lower than the maximum allowable limit established by the process $(110000 \mathrm{~Pa})$. The increment of the acetone feed flowrate causes the increment of the six parameters studied.
\end{abstract}

Keywords: Pressure drop; Design; Hairpins; Heat exchanger.

Nexo Revista Científica / Vol. 32, No. 02, pp. 150-165 / Diciembre 2019 


\section{NOMENCLATURA}

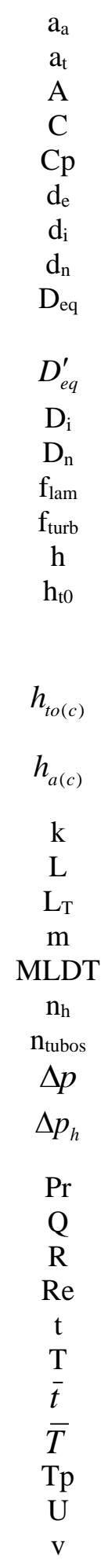
Área de flujo del ánulo Área de flujo del tubo interior Área total de transferencia de calor Constante Calor específico Diámetro exterior del tubo interior Diámetro interior del tubo interior Diámetro nominal del tubo interior Diámetro hidráulico equivalente del ánulo para la transferencia de calor

Diámetro hidráulico equivalente del ánulo para la caída de presión

Diámetro interior del ánulo

Diámetro nominal del ánulo

Fator de fricción para flujo laminar

Factor de fricción para flujo turbulento

Coeficiente pelicular de transferencia de calor

Coeficiente pelicular de transferencia de calor del fluido que circula por el tubo interior corregido con respecto a la superficie externa del tubo interior.

Coeficiente pelicular de transferencia de calor del fluido que circula por el tubo interior corregido con el factor de corrección de viscosidad

\section{$\mathrm{W} / \mathrm{m}^{2} \mathrm{~K}$}

Coeficiente pelicular de transferencia de calor del fluido que circula por el ánulo corregido con el factor de corrección de viscosidad

$\mathrm{W} / \mathrm{m}^{2} \mathrm{~K}$

Conductividad térmica

Longitud total del tubo

$\mathrm{W} / \mathrm{m} . \mathrm{K}$

Longitud del tubo

Caudal másico

Media Logarítmica de la Diferencia de Temperatura

Número de horquillas

Número total de tubos en serie

Caída de presión

$\mathrm{W} / \mathrm{m}^{2} \mathrm{~K}$

$\mathrm{W} / \mathrm{m}^{2} \mathrm{~K}$

$\mathrm{m}$

$\mathrm{m}$

$\mathrm{kg} / \mathrm{s}$

${ }^{\circ} \mathrm{C}$

$-$

Pa

Caída de presión del fluido que circula por el ánulo debido al paso de una horquilla hacia la otra

Número de Prandtl

Calor intercambiado

Factor de ensuciamiento

Número de Reynolds

Temperatura de la corriente fría

Temperatura de la corriente caliente

Temperatura media de la corriente fría

Temperatura media de la corriente caliente

Temperatura de la pared del tubo interior

Coeficiente global de transferencia de calor

Velocidad

$\mathrm{Pa}$

$\mathrm{W}$

K. $\mathrm{m}^{2} / \mathrm{W}$

${ }^{\circ} \mathrm{C}$

${ }^{\circ} \mathrm{C}$

${ }^{\circ} \mathrm{C}$

${ }^{\circ} \mathrm{C}$

${ }^{\circ} \mathrm{C}$

$\mathrm{W} / \mathrm{m}^{2} \mathrm{~K}$

$\mathrm{m} / \mathrm{s}$ 


\title{
A. Pérez Sánchez y E.J. Pérez Sánchez
}

\section{Símbolos griegos}

\section{$\rho$}

$\mu$
Densidad

Viscosidad $\mathrm{kg} / \mathrm{m}^{3}$

Pa.s

\section{Subíndices}

\author{
Entrada \\ Salida \\ Corriente caliente \\ Corriente fría \\ Del fluido que circula por el tubo interior \\ Del fluido que circula por el ánulo \\ A la temperatura de la pared del tubo interior
}

\section{INTRODUCCIÓN}

Los intercambiadores de calor son empleados en los procesos industriales para recuperar calor entre dos fluidos de proceso (Swamee et al., 2008). Entre los intercambiadores de calor más empleados en la industria moderna se encuentran los de doble tubo, debido fundamentalmente a su simplicidad de diseño y su capacidad para manejar fluidos a altas temperaturas y presiones (Mazumder y Mandal, 2016).

Un intercambiador de calor de doble tubo típico consiste de una tubería ubicada de forma concéntrica por dentro de otra tubería de mayor diámetro, conteniendo accesorios adecuados para dirigir el flujo desde una sección hacia la próxima.

En estos equipos, un fluido circula a través de la tubería interior (lado de los tubos), y el otro fluye a través del espacio anular (ánulo). La mayor aplicación que presentan los intercambiadores de calor de doble tubo consiste en el calentamiento o enfriamiento sensible de fluidos de proceso en donde se requieren pequeñas áreas de transferencia de calor (hasta $50 \mathrm{~m}^{2}$ ). La desventaja más notable que presentan radica en que son voluminosos y caros por unidad de área de transferencia de calor. Estos equipos también se denominan intercambiadores de calor de horquilla, y se emplean cuando una corriente es un gas, un líquido viscoso o presenta un pequeño volumen, y también bajo condiciones severas de ensuciamiento debido a su facilidad de limpieza y mantenimiento (Kakaç et al., 2012).

Varios autores han estudiado los intercambiadores de calor de doble tubo. En este sentido (Parekh y Chavda, 2014) llevaron a cabo el análisis experimental y exergético de un intercambiador de calor de doble tubo para un arreglo de flujo en paralelo. También (Swamee et al., 2008) formularon el diseño óptimo de un intercambiadores de calor de doble tubo mediante una programación geométrica con un grado simple de dificultad. Por otro lado (Soni y Khunt, 2015;Johnson et al., 2015;Dharmik et al., 2015) efectuaron el análisis de intercambiadores de calor de doble tubo mediante la Dinámica Computacional de Fluido. Además, (Hussein, 2015) realizaron una serie de experimentos para investigar el flujo turbulento y el comportamiento de la transferencia de calor en un intercambiador de calor de doble tubo equipado con deflectores semicirculares de disco. En otro estudio, (Mazumder y Mandal, 2016) proponen un modelo lineal aproximado simple para predecir las temperaturas de salida de un intercambiador de calor de doble tubo, considerándolo como de Black Box. Por último, (Ranjith y Shaji, 2016) analizaron el rendimiento de un intercambiador de calor de doble tubo equipado con cintas retorcidas y bajo condiciones de flujo turbulentas. Los resultados obtenidos en este estudio fueron validados utilizando correlaciones establecidas en la literatura. 


\section{A. Pérez Sánchez y E.J. Pérez Sánchez}

En el presente trabajo se lleva a cabo el diseño térmico de un intercambiador de calor de doble tubo para llevar a cabo el enfriamiento de acetona empleando etilenglicol como agente de enfriamiento. También se evalúa la influencia del incremento del caudal de la acetona sobre seis parámetros de diseño del intercambiador: Coeficiente global de transferencia de calor (U); área de transferencia de calor (A); Caída de presión de las corrientes de acetona y etilenglicol $\left(\Delta \mathrm{p}_{\mathrm{t}} \mathrm{y} \Delta \mathrm{p}_{\mathrm{a}}\right)$; calor intercambiado (Q) y Número total de horquillas necesarias $\left(\mathrm{n}_{\mathrm{h}}\right)$.

\section{MATERIALES Y MÉTODOS}

\subsection{Definición del problema}

Se necesitan enfriar a contracorriente $0,75 \mathrm{~kg} / \mathrm{s}$ de acetona desde 70 hasta $45{ }^{\circ} \mathrm{C}$ empleando etilenglicol a 5 ${ }^{\circ} \mathrm{C}$. La temperatura de salida del etilenglicol no deberá exceder los $25^{\circ} \mathrm{C}$. La caída de presión de ambas corrientes no deberá ser superior a $110000 \mathrm{~N} / \mathrm{m}^{2}$. Para ello se disponen de tubos con un diámetro de 1 pulg para el tubo interior, y de 2 pulg para el tubo exterior (ánulo), mientras que la longitud de los tubos $\left(\mathrm{L}_{\mathrm{T}}\right)$ no excederá los $3 \mathrm{~m}$. Diseñe un intercambiador de calor de doble tubo adecuado para este servicio.

\subsection{Metodología de cálculo a emplear}

Para efectuar el diseño térmico del intercambiador de calor de doble tubo se hará uso de la metodología publicada en (Cao, 2010), en la cual se obtiene como parámetro principal el número total de horquillas necesarias para cumplir con la demanda térmica del proceso en cuestión, asi como también las caídas de presión de ambos fluidos que circulan por el intercambiador de calor. A continuación, se definen las etapas o pasos a seguir para ejecutar el diseño de un equipo de este tipo.

\subsection{Número total de horquillas}

Paso 1. Definición de los datos iniciales requeridos:

- $\quad$ Temperatura de entrada y salida de la corriente caliente $\left(\mathrm{T}_{1}\right.$ y $\left.\mathrm{T}_{2}\right)$

- Temperatura de entrada y salida de la corriente fría $\left(t_{1}\right.$ y $\left.t_{2}\right)$

- Factores de ensuciamiento de ambas corrientes $\left(R_{C}\right.$ y $\left.R_{f}\right)$

- Caudal másico de al menos una corriente de fluido $\left(\mathrm{m}_{\mathrm{C}}\right.$ o $\left.\mathrm{m}_{\mathrm{f}}\right)$

- Caída de presión permisible para ambas corrientes $\left(\Delta \mathrm{p}_{\mathrm{C}}\right.$ y $\left.\Delta \mathrm{p}_{\mathrm{f}}\right)$

- Diámetro nominal del tubo interior $\left(\mathrm{d}_{\mathrm{n}}\right)$

- Diámetro nominal del tubo exterior (ánulo) $\left(\mathrm{D}_{\mathrm{n}}\right)$

- Longitud del tubo $\left(\mathrm{L}_{\mathrm{T}}\right)$

Paso 2. Definir los diámetros interiores y exteriores de ambas tuberías, de acuerdo con los diámetros nominales establecidos en el paso anterior.

Paso 3. Determinar la temperatura media de las corrientes involucradas

- Fluido caliente:

Nexo Revista Científica / Vol. 32, No. 02, pp. 150-165 / Diciembre 2019 


$$
\bar{T}=\frac{T_{1}+T_{2}}{2}
$$

- Fluido frío

$$
\bar{t}=\frac{t_{1}+t_{2}}{2}
$$

Paso 4. Determinar las propiedades físicas de ambos fluidos a la temperatura media calculada con anterioridad

Se deberán buscar la densidad $(\rho)$, viscosidad $(\mu)$, calor específico $(\mathrm{Cp})$ y conductividad térmica $(\mathrm{k})$ de ambos fluidos a la temperatura media determinada en el paso anterior. De esta manera, se recomienda confeccionar una tabla con los valores de estas cuatro variables una vez que hayan sido determinados.

\begin{tabular}{lcc}
\hline Variable & Valor & Unidades \\
\hline Densidad & $\mathrm{kg} / \mathrm{m}^{3}$ \\
Viscosidad & $\mathrm{Pa.s}$ \\
Calor especifico & $\mathrm{J} / \mathrm{kg} . \mathrm{K}$ \\
Conductividad térmica & $\mathrm{W} / \mathrm{m} . \mathrm{K}$ \\
\hline
\end{tabular}

Paso 5. Cálculo del calor intercambiado

El calor intercambiado se determina según los datos que se dispongan. De esta manera, si se disponen de todos los datos requeridos para la corriente caliente, el calor intercambiado se determinará según la siguiente ecuación:

$$
Q=m_{C} \cdot C p_{C} \cdot\left(T_{1}-T_{2}\right)
$$

Si por el contrario, se disponen de todos los datos requeridos para la corriente fría, entonces Q se determinará según la ecuación (4):

$$
Q=m_{f} \cdot C p_{f} \cdot\left(t_{2}-t_{1}\right)
$$

Paso 6. Cálculo del área de flujo del tubo interior:

El área de flujo del tubo interior (at) se determinará según la siguiente ecuación:

$$
a_{t}=\frac{\pi \cdot d_{i}^{2}}{4}
$$

Paso 7. Cálculo del área de flujo del ánulo:

El área de flujo del ánulo $\left(a_{a}\right)$ se calculará de acuerdo con la siguiente expresión:

$$
a_{a}=\pi \cdot \frac{D_{i}^{2}-d_{e}^{2}}{4}
$$

Nexo Revista Científica / Vol. 32, No. 02, pp. 150-165 / Diciembre 2019 


\section{A. Pérez Sánchez y E.J. Pérez Sánchez}

Paso 8. Ubicación de los fluidos dentro del intercambiador de calor

En la Tabla 1 se muestra las ecuaciones utilizadas para los pasos desde el 9 hasta el 15

Tabla 1. Ecuaciones empleadas para determinar las variables establecidas en los pasos 9-13

\begin{tabular}{|c|c|c|c|}
\hline Paso & Parámetro & Ecuación & $\begin{array}{l}\text { Ecuación } \\
\text { No. }\end{array}$ \\
\hline 9 & $\begin{array}{l}\text { Velocidad del fluido que circula por el tubo interior } \\
\text { (acetona) }\end{array}$ & $v_{t}=\frac{m}{\rho \cdot a_{t}}$ & (7) \\
\hline 10 & Velocidad del fluido que circula por el ánulo (etilenglicol) & $v_{a}=\frac{m}{\rho \cdot a_{a}}$ & (8) \\
\hline 11 & $\begin{array}{l}\text { Diámetro hidráulico equivalente para la transferencia de } \\
\text { calor }\end{array}$ & $D_{e q}=4 \cdot \frac{\pi \cdot\left(D_{i}^{2}-d_{e}^{2}\right)}{4 \cdot \pi \cdot d_{e}}$ & (9) \\
\hline 12 & $\begin{array}{l}\text { Número de Reynolds del fluido que circula por el tubo } \\
\text { interior (acetona) }\end{array}$ & $\operatorname{Re}_{t}=\frac{d_{i} \cdot v_{t} \cdot \rho_{t}}{\mu_{t}}$ & $(10)$ \\
\hline & $\begin{array}{l}\text { Número de Reynolds del fluido que circula por el ánulo } \\
\text { (etilenglicol) }\end{array}$ & $\operatorname{Re}_{a}=\frac{D_{e q} \cdot v \cdot \rho_{a}}{\mu_{a}}$ & (11) \\
\hline 13 & $\begin{array}{l}\text { Número de Prandtl del fluido que circula por el tubo interior } \\
\text { (acetona) }\end{array}$ & $\operatorname{Pr}_{t}=\frac{C p_{t} \cdot \mu_{t}}{k_{t}}$ & (12) \\
\hline & $\begin{array}{l}\text { Número de Prandtl del fluido que circula por el ánulo } \\
\text { (etilenglicol) }\end{array}$ & $\operatorname{Pr}_{a}=\frac{C p_{a} \cdot \mu_{a}}{k_{a}}$ & (13) \\
\hline
\end{tabular}

Fuente: Elaboración propia

Paso 14. Determinación de los coeficientes peliculares de intercambio de calor:

- Fluido que circula por el tubo interior:

Régimen laminar $\left(\operatorname{Re}_{\mathrm{t}}<2100\right)$ :

$$
h_{t}=1,86 \cdot \frac{k_{t}}{d_{i}} \cdot\left(\frac{\operatorname{Re}_{t} \cdot \operatorname{Pr}_{t}}{\frac{L_{T}}{d_{i}}}\right)^{0,33} \cdot\left(\frac{\mu_{t}}{\mu_{p t}}\right)^{0,14}
$$

Régimen de transición $\left(2100 \leq \operatorname{Re}_{\mathrm{t}} \leq 10000\right)$ :

Nexo Revista Científica / Vol. 32, No. 02, pp. 150-165 / Diciembre 2019 


$$
h_{t}=C p_{t} \cdot \rho_{t} \cdot v \cdot 0,116 \cdot\left(\frac{\operatorname{Re}_{t}^{0,66}-125}{\operatorname{Re}_{t}}\right) \cdot\left[1+\left(\frac{d_{i}}{L_{T}}\right)^{0,66}\right] \cdot \operatorname{Pr}_{t}^{-0,66} \cdot\left(\frac{\mu_{t}}{\mu_{p t}}\right)^{0,14}
$$

Régimen turbulento $\left(\mathrm{Re}_{\mathrm{t}}>10000\right)$ :

$$
h_{t}=\frac{k_{t}}{d_{i}} \cdot C \cdot \operatorname{Re}_{t}^{0,8} \cdot \operatorname{Pr}_{t}^{0,33} \cdot\left(\frac{\mu_{t}}{\mu_{p t}}\right)^{0,14}
$$

Dónde:

$$
\begin{aligned}
\mathrm{C} & =0,021 \text { para gases } \\
& =0,023 \text { para líquidos no viscosos } \\
& =0,027 \text { para líquidos viscosos }
\end{aligned}
$$

- Fluido que circula por el ánulo:

Régimen laminar $\left(\operatorname{Re}_{\mathrm{a}}<2100\right)$ :

$$
h_{a}=1,86 \cdot \frac{k_{a}}{D_{e q}} \cdot\left(\frac{\operatorname{Re}_{a} \cdot \operatorname{Pr}_{a}}{\frac{L_{t}}{D_{e q}}}\right)^{0,33} \cdot\left(\frac{\mu_{a}}{\mu_{p a}}\right)^{0,14}
$$

Régimen de transición $\left(2100 \leq \mathrm{Re}_{\mathrm{a}} \leq 10000\right)$ :

$$
h_{a}=C p_{a} \cdot \rho_{a} \cdot v \cdot 0,116 \cdot\left(\frac{\operatorname{Re}_{a}^{0,66}-125}{\operatorname{Re}_{a}}\right) \cdot\left[1+\left(\frac{D_{e q}}{L_{T}}\right)^{0,66}\right] \cdot \operatorname{Pr}_{a}^{-0,66} \cdot\left(\frac{\mu_{a}}{\mu_{p a}}\right)^{0,14}
$$

Régimen turbulento $\left(\operatorname{Re}_{\mathrm{a}}>10000\right)$ :

$$
h_{a}=\frac{k_{a}}{D_{e q}} \cdot C \cdot \operatorname{Re}_{a}^{0,8} \cdot \operatorname{Pr}_{a}^{0,33} \cdot\left(\frac{\mu_{a}}{\mu_{p a}}\right)^{0,14}
$$

Dónde:

$\mathrm{C}=0,021$ para gases

$=0,023$ para líquidos no viscosos

$=0,027$ para líquidos viscosos

Paso 15. Corrección del coeficiente pelicular de transferencia de calor del fluido que circula por el tubo interior con respecto a la superficie externa del tubo interior: 


$$
h_{t o}=h_{t} \cdot \frac{d_{i}}{d_{e}}
$$

Paso 16. Determinación de la temperatura de la pared del tubo interior:

Si el fluido que circula por el ánulo es el fluido caliente, la temperatura de la pared del tubo se determina según la siguiente expresión:

$$
h_{a} \cdot\left(\bar{T}-T_{p}\right)=h_{t o} \cdot\left(T_{p}-\bar{t}\right)
$$

Si el fluido caliente circula por el tubo interior, entonces:

$$
h_{t o} \cdot\left(\bar{T}-T_{p}\right)=h_{a} \cdot\left(T_{p}-\bar{t}\right)
$$

Paso 17. Búsqueda de la viscosidad de los fluidos que circulan tanto por el tubo interior como por el ánulo, a la temperatura de la pared del tubo $\left(\mathrm{T}_{\mathrm{p}}\right)$ determinada en el paso 16.

Buscar $\mu_{\mathrm{pt}} \mathrm{y} \mu_{\mathrm{pa}}$ a $\mathrm{T}_{\mathrm{p}}$

La Tabla 2 muestra las ecuaciones empleadas para determinar las variables incluidas en los pasos 18-25

Tabla 2. Ecuaciones empleadas para determinar las variables incluidas en los pasos 18-25.

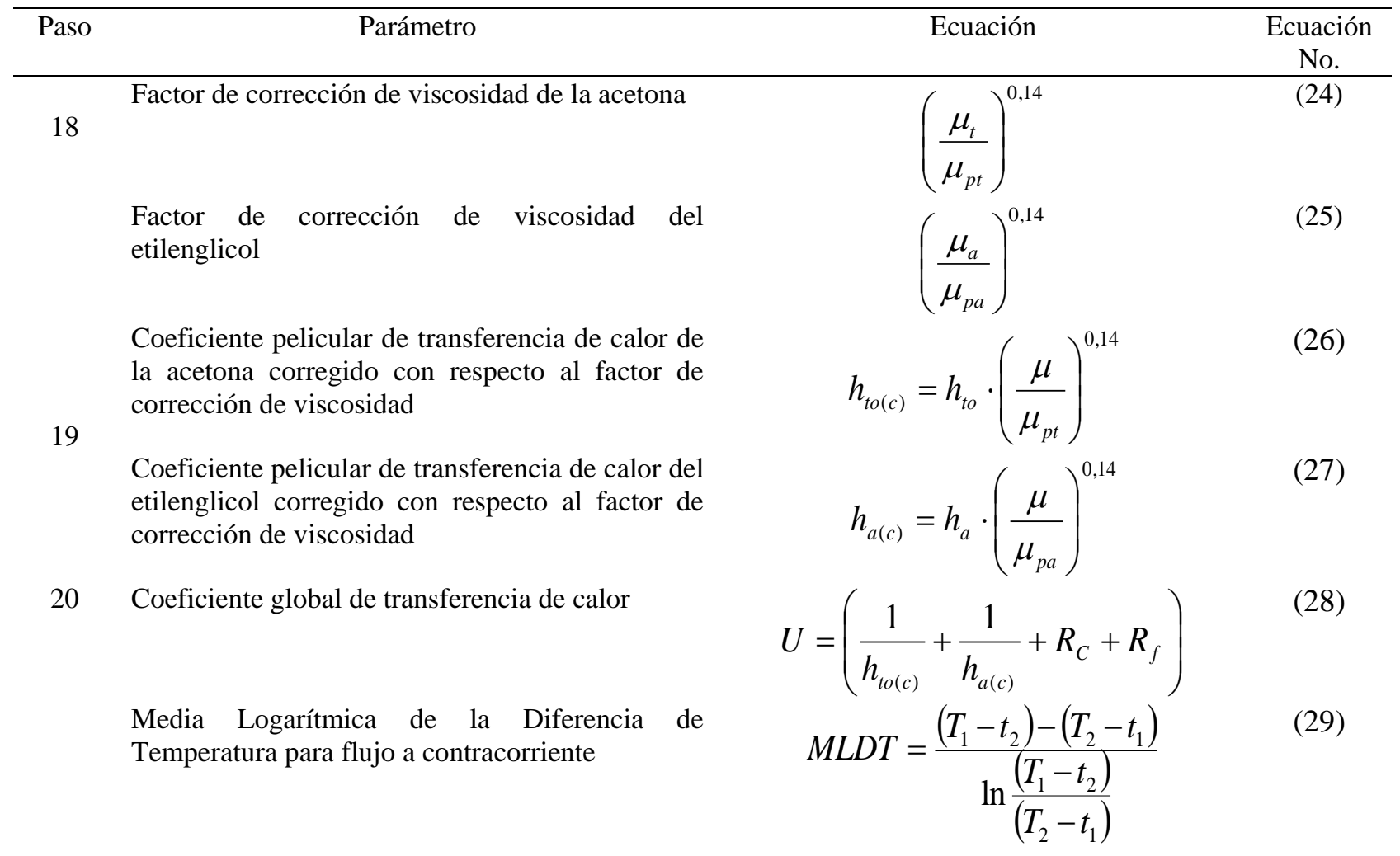




\section{A. Pérez Sánchez y E.J. Pérez Sánchez}

21 Media Logarítmica de la Diferencia de

Temperatura para flujo en paralelo

$$
M L D T=\frac{\left(T_{1}-t_{1}\right)-\left(T_{2}-t_{2}\right)}{\ln \frac{\left(T_{1}-t_{1}\right)}{\left(T_{2}-t_{2}\right)}}
$$

22 Área de transferencia de calor requerida

$$
A=\frac{Q}{U \cdot M L D T}
$$

23 Longitud total del tubo

$$
L=\frac{A}{\pi \cdot d_{e}}
$$

$24 \quad$ Número total de tubos en serie

$$
n_{\text {tubos }}=\frac{L}{L_{T}}
$$

$25 \quad$ Número de horquillas

$$
n_{h}=\frac{n_{\text {tubos }}}{2}
$$

Fuente: Elaboración propia

\subsection{Caída de presión}

La Tabla 3 expone las ecuaciones empleadas para determinar los parámetros establecidos en los pasos 2631, con la finalidad de determinar la caída de presión de ambos fluidos.

Tabla 3. Ecuaciones empleadas para en los pasos 26-31.

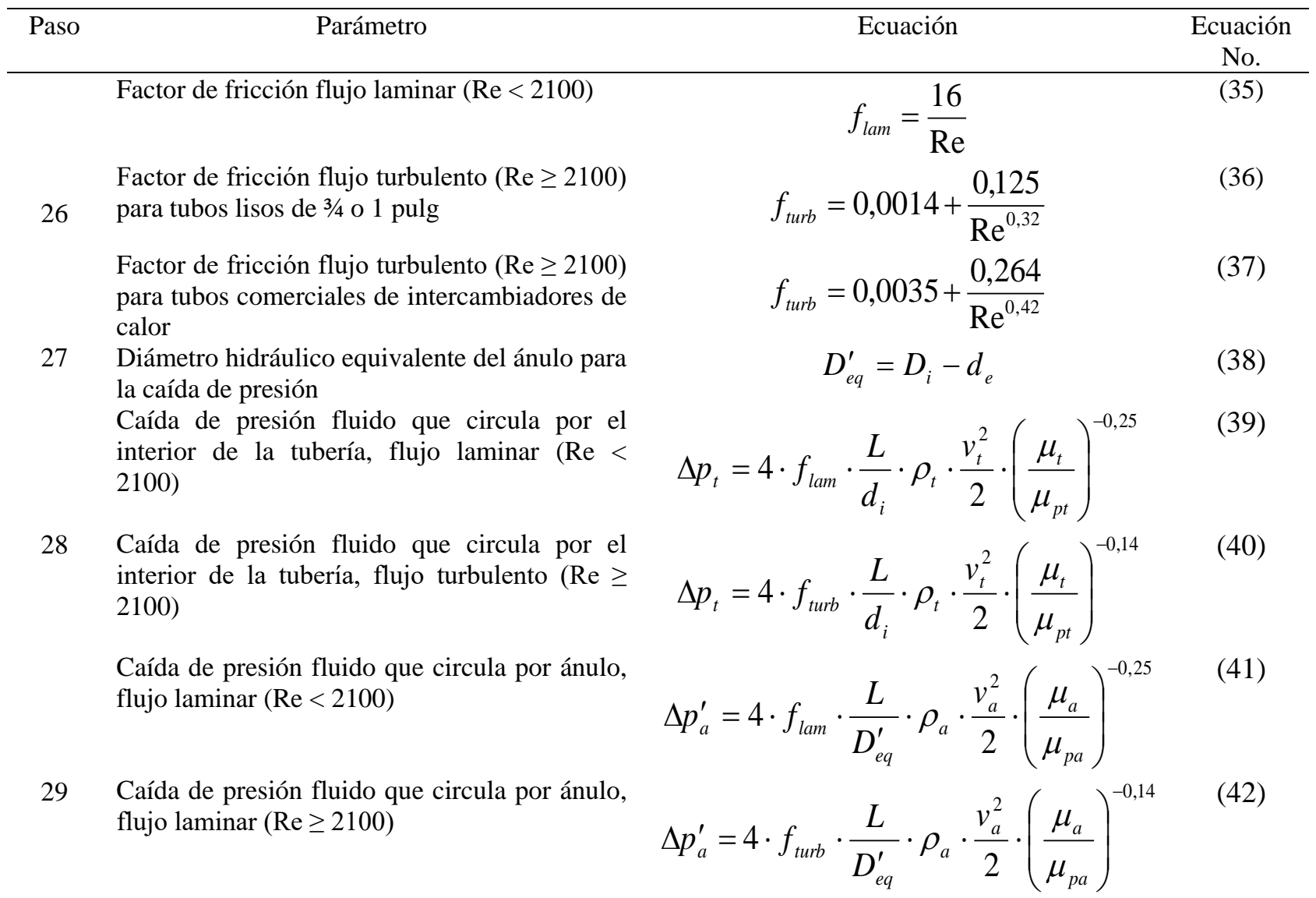




\section{A. Pérez Sánchez y E.J. Pérez Sánchez}

30

Caída de presión del fluido que circula por el ánulo debido al paso de una horquilla hacia la otra

31 Caída de presión total del fluido que circula por el ánulo

$$
\begin{gathered}
\Delta p_{h}=\frac{n_{\text {tubos }}}{2} \cdot \frac{\rho_{a} \cdot v_{a}^{2}}{2} \\
\Delta p_{a}=\Delta p_{a}^{\prime}+\Delta p_{h}
\end{gathered}
$$

Fuente: Elaboración propia

2.5. Influencia del incremento del caudal de la acetona sobre seis parámetros de diseño del intercambiador

Se evaluó la influencia del incremento del caudal másico de alimentación de la acetona sobre seis parámetros de diseño del intercambiador de calor de doble tubo, los cuales son: coeficiente global de transferencia de calor (U); área de transferencia de calor (A); caída de presión de las corrientes de acetona y etilenglicol $\left(\Delta \mathrm{p}_{\mathrm{t}}\right.$ y $\left.\Delta \mathrm{p}_{\mathrm{a}}\right)$; calor intercambiado (Q) y número total de horquillas necesarias $\left(\mathrm{n}_{\mathrm{h}}\right)$. Se obtendrán gráficos que relacionen estas variables, para lograr una mejor comprensión del estudio. De esta manera, el caudal de alimentación de la acetona se variará entre $0,5-3,0 \mathrm{~kg} / \mathrm{s}$.

\section{RESULTADOS Y DISCUSIÓN}

\subsection{Número total de horquillas}

A continuación, se muestran los resultados obtenidos para cada uno de los pasos involucrados en el diseño del intercambiador de calor de doble tubo hasta determinar el número total de horquillas necesarias para cumplir con la demanda térmica del sistema. En la Tabla 4 se exponen los parámetros iniciales definidos por el problema de acuerdo con el paso 1.

Tabla 4. Parámetros iniciales definidos en el paso 1.

\begin{tabular}{lccc}
\hline \multicolumn{1}{c}{ Parámetro } & Símbolo & Valor & Unidades \\
\hline Temperatura de entrada acetona & $\mathrm{T}_{1}$ & 70 & ${ }^{\circ} \mathrm{C}$ \\
Temperatura de salida acetona & $\mathrm{T}_{2}$ & 45 & ${ }^{\circ} \mathrm{C}$ \\
Caudal másico acetona & $\mathrm{m}_{\mathrm{C}}$ & 0,75 & $\mathrm{~kg} / \mathrm{s}$ \\
Factor de ensuciamiento acetona ${ }^{\S}$ & $\mathrm{R}_{\mathrm{C}}$ & 0,0002 & $\mathrm{~m}^{20} \mathrm{C} / \mathrm{W}$ \\
Caída de presión permisible acetona & $\Delta \mathrm{P}_{\mathrm{C}}$ & 110000 & $\mathrm{~Pa}$ \\
Temperatura de entrada etilenglicol & $\mathrm{t}_{1}$ & 5 & ${ }^{\circ} \mathrm{C}$ \\
Temperatura de salida etilenglicol & $\mathrm{t}_{2}$ & 25 & ${ }^{\circ} \mathrm{C}$ \\
Factor de ensuciamiento etilenglicol & $\mathrm{R}_{\mathrm{f}}$ & 0,0002 & $\mathrm{~m}^{20} \mathrm{C} / \mathrm{W}$ \\
Caída de presión permisible etilenglicol & $\Delta \mathrm{P}_{\mathrm{f}}$ & 110000 & $\mathrm{~Pa}$ \\
Diámetro nominal tubo interior & $\mathrm{d}_{\mathrm{n}}$ & 1 & $\mathrm{pulg}$ \\
Diámetro nominal ánulo & $\mathrm{D}_{\mathrm{n}}$ & 2 & $\mathrm{pulg}$ \\
Longitud del tubo & $\mathrm{L}_{\mathrm{T}}$ & 3 & $\mathrm{~m}$ \\
\hline
\end{tabular}

$\S$ Tomado de (Sinnott, 2005)

Fuente: Elaboración propia

Paso 2. Definición de los diámetros interiores y exteriores para ambos tubos.

Según (Perry, 2008), para un tubo de diámetro nominal igual a 1 pulg, los diámetros interior y exterior para este tubo serán:

- Diámetro interior (di): 0,02664 m.

- Diámetro exterior (de): 0,03340 m. 


\section{A. Pérez Sánchez y E.J. Pérez Sánchez}

Por su parte, para un tubo de diámetro nominal de 2 pulg, el diámetro interior (Di) de este tubo será de 0,05250 $\mathrm{m}$ de acuerdo con (Perry, 2008).

Paso 3. Cálculo de la temperatura media de ambas corrientes:

- Acetona:

$$
\bar{T}=\frac{T_{1}+T_{2}}{2}=\frac{70+45}{2}=57,5^{\circ} \mathrm{C}
$$

- Fluido frío

$$
\bar{t}=\frac{t_{1}+t_{2}}{2}=\frac{5+25}{2}=15^{\circ} \mathrm{C}
$$

Paso 4. Propiedades físicas de ambos fluidos:

Según (Perry, 2008), tanto la acetona como el etilenglicol presentan los siguientes valores de las propiedades físicas (Tabla 5):

Tabla 5. Propiedades físicas de ambos fluidos a la temperatura media determinada en el Paso 3

\begin{tabular}{lcc}
\hline Propiedad & Acetona & Etilenglicol \\
\hline Calor específico [J/kg.K] & 2289,97 & 2353,05 \\
Densidad $\left[\mathrm{kg} / \mathrm{m}^{3}\right]$ & 748,26 & 1117,21 \\
Viscosidad [Pa.s] & 0,0002329 & 0,02606 \\
Conductividad térmica [W/m.K] & 0,147 & 0,252 \\
\hline Fuente: Elaboración propia & &
\end{tabular}

Paso 5. Calor intercambiado:

Se utilizará la corriente de acetona por ser la que presenta los datos completos. De esta manera:

$$
Q=m_{C} \cdot C p_{C} \cdot\left(T_{1}-T_{2}\right)=42936,94 W
$$

A continuación, se determinará el flujo másico del etilenglicol necesario para cumplir con la demanda térmica del proceso.

Partiendo de:

$$
Q=m_{f} \cdot C p_{f} \cdot\left(t_{2}-t_{1}\right)
$$

Se despeja y calcula $\mathrm{m}_{\mathrm{f}}$ :

$$
m_{f}=\frac{Q}{C p_{f} \cdot\left(t_{2}-t_{1}\right)}=0,91 \frac{\mathrm{kg}}{\mathrm{s}}
$$

Paso 6. Cálculo del área de flujo del tubo interior: 


$$
a_{t}=\frac{\pi \cdot d_{i}^{2}}{4}=0,000557 \mathrm{~m}^{2}
$$

Paso 7. Cálculo del área de flujo del ánulo:

$$
a_{a}=\pi \cdot \frac{D_{i}^{2}-d_{e}^{2}}{4}=0,00129 m^{2}
$$

Paso 8. Ubicación de los fluidos dentro del intercambiador de calor

Siguiendo las recomendaciones y sugerencias publicadas en (Kern, 1999)(Sinnott, 2005) con respecto a la ubicación de fluidos en intercambiadores de calor, se propone ubicar el etilenglicol por el ánulo y la acetona por el tubo interior.

La Tabla 6 muestra los resultados de los parámetros determinados en los pasos 9-16.

\begin{tabular}{|c|c|c|c|c|}
\hline Paso & Parámetro & Símbolo & Valor & Unidades \\
\hline 9 & $\begin{array}{l}\text { Velocidad del fluido que circula por el tubo interior } \\
\text { (acetona) }\end{array}$ & $\mathrm{V}_{\mathrm{t}}$ & 1,80 & $\mathrm{~m} / \mathrm{s}$ \\
\hline 10 & $\begin{array}{l}\text { Velocidad del fluido que circula por el ánulo } \\
\text { (etilenglicol) }\end{array}$ & $\mathrm{v}_{\mathrm{a}}$ & 0,63 & $\mathrm{~m} / \mathrm{s}$ \\
\hline \multirow[t]{2}{*}{11} & $\begin{array}{l}\text { Diámetro hidráulico equivalente para la transferencia de } \\
\text { calor }\end{array}$ & $\mathrm{D}_{\mathrm{eq}}$ & 0,049 & $\mathrm{~m}$ \\
\hline & Número de Reynolds de la acetona & $\mathrm{Re}_{\mathrm{t}}$ & 154059,9 & - \\
\hline \multirow[t]{2}{*}{12} & Número de Reynolds del etilenglicol & $\operatorname{Re}_{\mathrm{a}}$ & 1323,41 & - \\
\hline & Número de Prandtl de la acetona & $\operatorname{Pr}_{t}$ & 3,63 & - \\
\hline 13 & Número de Prandtl del etilenglicol & $\mathrm{Pr}_{\mathrm{a}}$ & 243,33 & - \\
\hline \multirow[t]{2}{*}{14} & $\begin{array}{l}\text { Coeficiente pelicular de transferencia de calor de la } \\
\text { acetona }\end{array}$ & $h_{t}$ & 2743,8 & $\mathrm{~W} / \mathrm{m}^{2} \mathrm{~K}$ \\
\hline & $\begin{array}{l}\text { Coeficiente pelicular de transferencia de calor del } \\
\text { etilenglicol }\end{array}$ & $\mathrm{h}_{\mathrm{a}}$ & 161,58 & $\mathrm{~W} / \mathrm{m}^{2} \mathrm{~K}$ \\
\hline 15 & $\begin{array}{l}\text { Coeficiente pelicular de transferencia de calor de la } \\
\text { acetona corregido con respecto a la superficie externa del } \\
\text { tubo interior }\end{array}$ & $h_{t 0}$ & 2188,47 & $\mathrm{~W} / \mathrm{m}^{2} \mathrm{~K}$ \\
\hline 16 & Temperatura de la pared del tubo & $T_{p}$ & 54,6 & ${ }^{\circ} \mathrm{C}$ \\
\hline
\end{tabular}

Tabla 6. Resultados de los parámetros determinados en los pasos 9-16

Paso 17. Búsqueda de la viscosidad de los fluidos que circulan tanto por el tubo interior como por el ánulo, a la temperatura de la pared del tubo $\left(\mathrm{T}_{\mathrm{p}}\right)$ de $54,6^{\circ} \mathrm{C}$.

Acetona $\left(\mu_{\mathrm{pt}}\right)=0,0002381$ Pa.s

Etilenglicol $\left(\mu_{\mathrm{pa}}\right)=0,005978$ Pa.s

La Tabla 7 muestra los resultados de los parámetros determinados en los pasos 18-25. 


\section{A. Pérez Sánchez y E.J. Pérez Sánchez}

Tabla 7. Resultados de los parámetros determinados en los pasos 18-25

\begin{tabular}{|c|c|c|c|c|}
\hline Paso & Parámetro & Símbolo & Valor & Unidades \\
\hline \multirow{5}{*}{18} & Factor de corrección de viscosidad de la acetona & 0,14 & & \\
\hline & & $\mu_{t}$ & 0,99 & - \\
\hline & Factor de corrección de viscosidad del etilenglicol & 0, & & \\
\hline & & $\mu_{a}$ & 1,22 & - \\
\hline & $\begin{array}{l}\text { Coeficiente pelicular de transferencia de calor de la } \\
\text { acetona corregido con respecto al factor de corrección de } \\
\text { viscosidad }\end{array}$ & $\mathrm{h}_{\mathrm{t} 0(\mathrm{c})}$ & 2166,58 & $\mathrm{~W} / \mathrm{m}^{2} \mathrm{~K}$ \\
\hline 19 & $\begin{array}{l}\text { Coeficiente pelicular de transferencia de calor del } \\
\text { etilenglicol corregido con respecto al factor de corrección } \\
\text { de viscosidad }\end{array}$ & $\mathrm{h}_{\mathrm{a}(\mathrm{c})}$ & 197,13 & $\mathrm{~W} / \mathrm{m}^{2} \mathrm{~K}$ \\
\hline 20 & Coeficiente global de transferencia de calor & $\mathrm{U}$ & 168,59 & $\mathrm{~W} / \mathrm{m}^{2} \mathrm{~K}$ \\
\hline 21 & Media Logarítmica de la Diferencia de Temperatura & MLDT & 42,44 & ${ }^{\circ} \mathrm{C}$ \\
\hline 22 & Área de transferencia de calor requerida & A & 6,0 & $\mathrm{~m}^{2}$ \\
\hline 23 & Longitud total del tubo & $\mathrm{L}$ & 57,2 & $\mathrm{~m}$ \\
\hline 24 & Número total de tubos en serie & $\mathrm{n}_{\text {tubos }}$ & 19,1 & - \\
\hline 25 & Número de horquillas & $\mathrm{n}_{\mathrm{h}}$ & 10 & - \\
\hline
\end{tabular}

3.2. Caída de presión

La Tabla 8 exhibe los resultados obtenidos para los parámetros determinados en los pasos 26-31

Tabla 8. Valores de los parámetros determinados en los pasos 26-31.

\begin{tabular}{clccc}
\hline Paso & \multicolumn{1}{c}{ Parámetro } & Símbolo & Valor & Unidades \\
\hline 26 & Factor de fricción de la acetona & $\mathrm{f}_{\text {turb }}$ & 0,00525 & - \\
& Factor de fricción del etilenglicol & $\mathrm{f}_{\mathrm{lam}}$ & 0,0121 & - \\
27 & $\begin{array}{l}\text { Diámetro hidráulico equivalente del ánulo para la } \\
\text { caída de presión }\end{array}$ & $D_{e q}^{\prime}$ & 0,0191 & $\mathrm{~m}$ \\
28 & $\begin{array}{c}\text { Caída de presión de la acetona } \\
29\end{array}$ & $\Delta \mathrm{p}_{\mathrm{t}}$ & 54826,79 & $\mathrm{~Pa}$ \\
30 & $\begin{array}{l}\text { Caída de presión del etilenglicol } \\
\text { Caída de presión del etilenglicol debido al paso }\end{array}$ & $\Delta \mathrm{p}_{\mathrm{a}}$ & 22238,19 & $\mathrm{~Pa}$ \\
& $\begin{array}{l}\text { de una horquilla hacia la otra } \\
31\end{array}$ & 2117,33 & $\mathrm{~Pa}$ \\
& $\begin{array}{l}\text { Caída de presión total del etilenglicol debido a su } \\
\text { paso por el ánulo }\end{array}$ & $\Delta \mathrm{p}_{\mathrm{a}}$ & 24355,52 & $\mathrm{~Pa}$ \\
\hline
\end{tabular}

Fuente: Elaboración propia

Según se puede observar en la Tabla 6, la corriente de etilenglicol se encuentra en régimen de flujo laminar, ya que el número de Reynolds es menor que 2100 (1 323,41). También se puede notar en esta tabla que el coeficiente pelicular de transferencia de calor de la acetona es casi 17 veces superior al coeficiente pelicular del etilenglicol. De forma general, se obtuvo un coeficiente global de transferencia de calor de 168,59 W/m ${ }^{2} \mathrm{~K}$, el cual puede considerarse de bajo (Cao, 2010), un área de transferencia de calor de $6,0 \mathrm{~m}^{2}$, un valor del número total de tubos en serie de 19,1 , y un número total de horquillas necesarias igual a 10 (Tabla 7).

Por otra parte, de acuerdo con los resultados mostrados en la Tabla 8, tanto la caída de presión calculada para la corriente de acetona (54 826,79 Pa) como para la del etilenglicol (24 355,52 Pa) se encuentran por debajo de la caída de presión máxima permisible establecida por el proceso para ambas corrientes (110

Nexo Revista Científica / Vol. 32, No. 02, pp. 150-165 / Diciembre 2019 


\section{A. Pérez Sánchez y E.J. Pérez Sánchez}

$000 \mathrm{~Pa}$ ), lo cual indica un comportamiento favorable en este sentido. Vale destacar que la caída de presión de la acetona es aproximadamente 2,25 veces superior a la del etilenglicol, lo cual se debe fundamentalmente a la mayor velocidad que presenta la corriente de acetona $(1,80 \mathrm{~m} / \mathrm{s})$ en comparación con la velocidad de la corriente de etilenglicol $(0,63 \mathrm{~m} / \mathrm{s})$.

3.3. Resultados de la influencia del incremento del caudal de alimentación de la acetona sobre 6 parámetros de diseño del intercambiador

La Figura 1 muestra los gráficos obtenidos que relacionan las 6 variables de diseño del intercambiador de calor de doble tubo con el incremento del caudal de alimentación de la acetona.

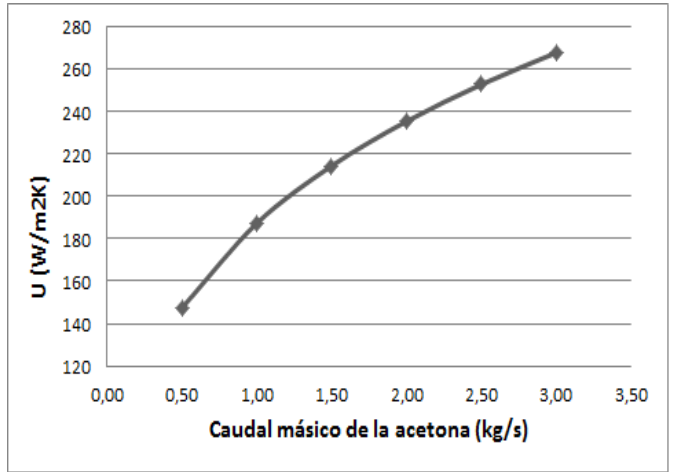

(a)

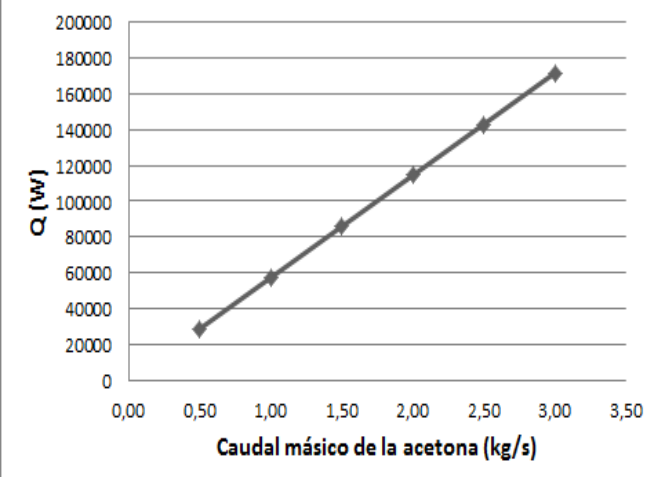

(c)

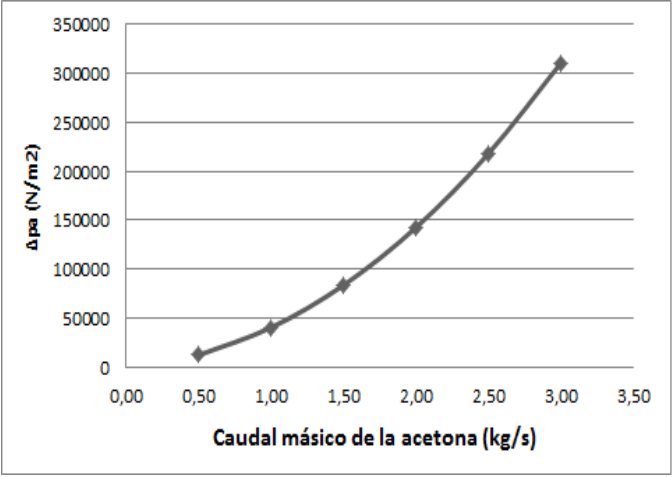

(e)

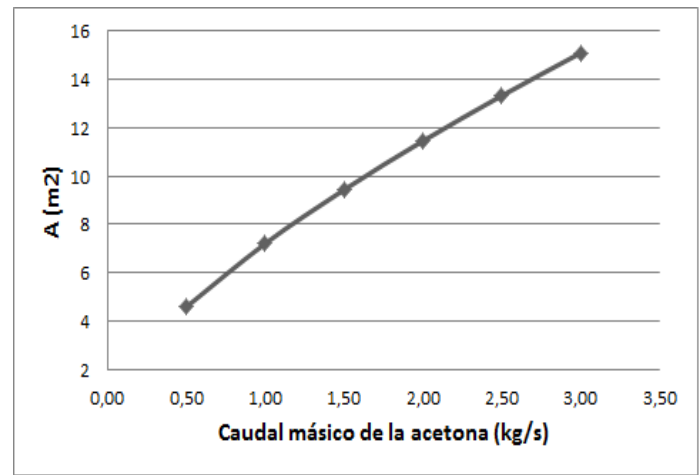

(b)

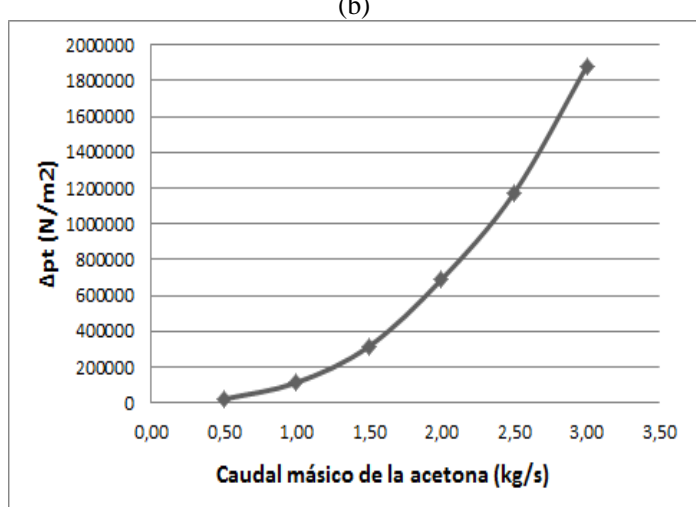

(d)

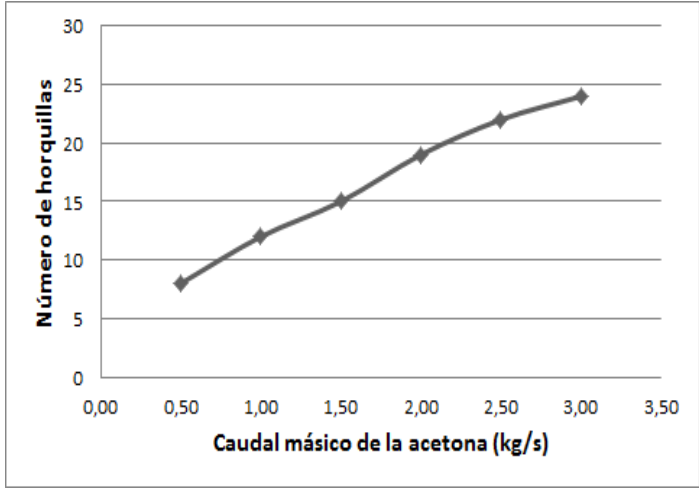

(f)

Figura 1. Influencia del incremento del caudal de alimentación sobre seis parámetros de diseño del intercambiador de calor de doble tubo. (a) Coeficiente global de transferencia de calor. (b) Área total de transferencia de calor. (c) Calor intercambiado. (d) Caída de presión de la acetona. (e) Caída de presión del etilenglicol. (f) Número de horquillas. Fuente: Elaboración propia 


\section{A. Pérez Sánchez y E.J. Pérez Sánchez}

Como bien se puede observar en la Fig. 2, todos los parámetros de diseño evaluados se incrementan con un aumento del caudal másico de la acetona. Esto se debe a que a mayor caudal de la acetona, mayor será el calor intercambiado (Q) según la ecuación (3), y mayores serán además la velocidad lineal $\left(\mathrm{v}_{\mathrm{t}}\right)$ [ecuación (7)], el número de Reynolds $\left(\mathrm{Re}_{\mathrm{t}}\right.$ ) [ecuación (10)]; el coeficiente pelicular de transferencia de calor $\left(h_{t}\right)$ [ecuación 16)]; el área de transferencia de calor (A) [ecuación (31)]; la longitud total del tubo (L) [ecuación (32)]; el número total de tubos en serie ( $n_{\text {tubos }}$ ) [ecuación (33)] y con ello finalmente el número total de horquillas $\left(\mathrm{n}_{\mathrm{h}}\right)$ [ecuación (34)]. Es decir, existe una relación directamente proporcional entre la variable independiente (caudal de alimentación de la acetona) y las seis variables dependientes analizadas.

En el caso de la caída de presión de la acetona, esta presenta un comportamiento exponencial en donde se supera el límite máximo permisible por el proceso (110 $000 \mathrm{~Pa}$ ) cuando el caudal de la acetona es superior a $0,9855 \mathrm{~kg} / \mathrm{s}$. En el caso de la caída de presión del etilenglicol, el grafico obtenido también presenta un comportamiento exponencial, superándose el límite máximo permisible por el proceso para esta sustancia (110 $000 \mathrm{~Pa}$ ) cuando el caudal de la acetona supera los 1,7198 kg/s. Los restantes parámetros presentan un comportamiento lineal o cercano a este.

\section{CONCLUSIONES}

El intercambiador de calor de doble tubo deberá tener un área total de transferencia de calor de $6,0 \mathrm{~m}^{2} \mathrm{y}$ un número total de horquillas de 10 para cumplir con la demanda térmica del proceso.

Tanto la caída de presión de la acetona (54 826,79 Pa) como la del etilenglicol (24 355,52 Pa) se encuentran por debajo del límite máximo permisible establecido por el proceso (110 $000 \mathrm{~Pa})$.

La caída de presión de la acetona superará el límite máximo permisible establecido por el proceso (110 $000 \mathrm{~Pa}$ ) cuando el caudal de alimentación de la acetona supere los $0,9855 \mathrm{~kg} / \mathrm{s}$.

La caída de presión del etilenglicol superará el límite máximo permisible establecido por el proceso (110 $000 \mathrm{~Pa}$ ) cuando el caudal de alimentación de la acetona supere $\operatorname{los} 1,7198 \mathrm{~kg} / \mathrm{s}$.

Los seis parámetros evaluados presentan una tendencia creciente al incrementarse el caudal másico de alimentación de la acetona.

\section{REFERENCIAS}

Cao, E. (2010). Heat transfer in process engineering. New York: McGraw-Hill.

Dharmik, P., Dhiman, V. D., Patel, J. J., and Engineer, R. (2015). CFD analysis of triple concentric tube heat exchanger. University Journal of Research, 1 (1), 30-44.

Hussein, S. A. A. (2015). Experimental Investigation of Double Pipe Heat Exchanger by using Semi Circular Disc Baffles. International Journal of Computer Applications, 115 (4), 13-17.

Johnson, J., Anzar, A., Shani, A., Rahiman, H., Hameed, H., and Nithin, V. S. (2015). CFD Analysis of Double Pipe Heat Exchanger. International Journal of Science, Engineering and Technology Research, 4 (5), 1283-1286.

Kakaç, S., Liu, H., and Pramuanjaroenkij, A. (2012). Heat Exchangers. Selection, Rating, and Thermal Design. Boca Raton: CRC Press. 


\section{A. Pérez Sánchez y E.J. Pérez Sánchez}

Kern, D. Q. (1999). Procesos de Transferencia de Calor. Mexico D.F: Compañía Editorial Continental, S.A. de C.V.

Mazumder, A., and Mandal, B. K. (2016). Numerical Modeling and Simulation of a Double Tube Heat Exchanger Adopting a Black Box Approach. International Journal of Engineering Research and Applications, 6 (4), 35-41.

Parekh, P. P., \& Chavda, N. K. (2014). Experimental and Exergy Analysis of a Double Pipe Heat Exchanger for Parallel Flow Arrangement. International Journal of Engineering Research and Applications, 4 (7), 9-13.

Perry, R. H. (2008). Chemical Engineers' Handbook (8th ed.). New York: McGraw-Hill.

Ranjith S., and Shaji, K. (2016). Numerical analysis on a double pipe heat exchanger with twisted tape induced swirl flow on both sides. Procedia Technology, 24, 436 - 443.

Sinnott, R. K. (2005). Chemical Engineering Design (4th ed. Vol. 6). Oxford: Elsevier ButterworthHeinemann.

Soni, J. R., and Khunt, J. B. (2015). CFD Analysis and Performance Evaluation of Concentric Tube in Tube Heat Exchanger. International Journal for Innovative Research in Science \& Technology, 2 (1), 1821.

Swamee, P. K., Aggarwal, N., \& Aggarwal, V. (2008). Optimum design of double pipe heat exchanger. International Journal of Heat and Mass Transfer, 51, 2260-2266.

\section{SEMBLANZA DE LOS AUTORES}

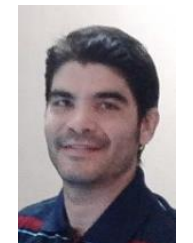

Amaury Pérez Sánchez: Obtuvo el grado de Ingeniero Químico en la Universidad de Camagüey, Cuba, donde actualmente es profesor instructor e investigador auxiliar. En estos momentos se encuentra estudiando un Máster en Biotecnología en el centro de Ingeniería Genética y Biotecnología de Camagüey. Trabaja en líneas de investigación vinculadas fundamentalmente con el diseño de equipos de transferencia de calor y masa, simulación y optimización de procesos y operaciones de la industria química mediante el empleo de simuladores, y la evaluación técnico-económica de procesos y plantas biotecnológicas.

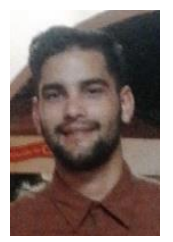

Eddy Javier Pérez Sánchez: Obtuvo el grado de Ingeniero Químico en la Universidad de Camagüey, Cuba. Se desempeña profesionalmente en la Empresa de Cítricos Ceballos, en la provincia de Ciego de Ávila, Cuba, donde atiende la línea de procesamiento de tomate y guayaba para la producción de néctares y pastas. Trabaja en líneas de investigación relacionadas con el diseño, evaluación y/o operación de equipos de transferencia de calor y masa.

Nexo Revista Científica / Vol. 32, No. 02, pp. 150-165 / Diciembre 2019 